\title{
Knee joint function and the energy cost of level walking in soccer players
}

\author{
L J Tofts, C S Stanley, T G Barnett, J G Logan
}

\begin{abstract}
Objectives-To study self reported knee joint problems and the energy costs of level walking in soccer players.

Methods-Seventeen soccer players and twelve control subjects between 18 and 27 years old participated in the study. A questionnaire was used to establish the amount of participation in soccer and the frequency and extent of knee injuries. The physiological cost index (PCI) was used as an index of the energy costs of level walking.

Results-Soccer players had a significantly higher PCI than control subjects (p $=0.0001)$. Control subjects had a mean (SD) PCI of $0.23(0.06)$ beats $/ \mathrm{m}$ and soccer players had a mean PCI of $0.42(0.12)$ beats $/ \mathrm{m}$. Some $82 \%$ of the soccer players experienced knee joint problems, whereas only $25 \%$ of the control group had problems.

Conclusions-This study shows that college soccer players have a higher rate of self reported knee problems and higher energy costs of level walking than people who do not play soccer.

(Br F Sports Med 1998;32:130-133)
\end{abstract}

Keywords: knee joint physiology; soccer physiology; physiological cost index; soccer injuries

Soccer players sustain acute musculoskeletal injuries but the long term effects of repeated injury and overuse are not well established. Approximately two thirds of soccer injuries are traumatic and one third are due to overuse, with injury to the lower limb accounting for $60-90 \%$ of all injuries. ${ }^{1}$

In this study we aim to determine whether soccer players and non-players have comparable physiological cost indices (PCIs) for level walking and rates of self reported knee joint problems. We also analysed whether the standard of the team or the frequency of playing soccer either this season or at school affected PCI data.

The standard physiological measurement of energy expenditure is oxygen consumption (in $\mathrm{ml} / \mathrm{kg} /$ minute). PCI has a linear relation with oxygen consumption over a wide range of walking speeds $^{23}$ and therefore is a valid indicator of energy expenditure during level walking.

\section{Methods}

Soccer players were recruited from college teams, and control subjects were recruited from the college population. Potential subjects were excluded if they reported a family history of rheumatoid arthritis, any cardiac, pulmonary, or neuromuscular pathology, and if they had sustained any serious injury to legs or pelvis that was not sports related.

Ethical approval was obtained from the Ethics Committee for Human Research, East London and the City Health Authority. All subjects provided written and informed consent to participate in the study.

Age, height, and weight were recorded. The study was carried out during January and February, in the middle of the soccer season.

MEASUREMENT OF PCI

Heart rates were determined from an ambulatory electrocardiogram recorded using a radiotelemetry system (MTR8S) supplied by MIE (Medical Research, Leeds, UK). Self selected walking speed (WS) and walking heart rate (WHR) were measured simultaneously as the subjects walked at their natural pace around a marked rectangular $20 \mathrm{~m}$ circuit $(8 \mathrm{~m} \times 2 \mathrm{~m})$. The number of laps, time taken, and heart rate were recorded. Resting and walking heart rate were measured every minute, and when three equal values were obtained sequentially the rate was deemed to have stabilised and was recorded. Resting heart rate (RHR) was measured after the floor walk, as anticipation of exercise may raise it as the result of sympathetic effects. As the method of recording the rate depends on obtaining three sequential equal values, the rate could not have been recorded before the subject recovered from exercise. The PCI was calculated using the heart rate and walking speed information in the following equation:

$$
\mathrm{PCI}=\frac{\mathrm{WHR}-\mathrm{RHR}}{\mathrm{WS}}
$$

The units of PCI are beats $/ \mathrm{m}$ travelled (beats $/ \mathrm{m}$ ).

THE QUESTIONNAIRE

The questionnaire is reproduced as fig 1. Subjects were questioned verbally by a researcher who filled in the form. If they reported knee joint problems, they were questioned further

Table 1 Walking (WHR) and resting (RHR) heart rate, walking speed (WS) and physiological cost index (PCI) for soccer players and control subjects (values are mean (SD))

\begin{tabular}{lll}
\hline & Soccer players & Control subjects \\
\hline RHR (beats/min) & $68.2(13.1)$ & $78.5(14.7)$ \\
WHR (beats/min) & $94.9(11.8)$ & $94.2(13.6)$ \\
CHR (beats/min) & $26.7(6.6)$ & $15.7(3.5)$ \\
WS (m/min) & $64.9(9.3)$ & $67.6(8.1)$ \\
PCI (beats/m) & $0.42(0.12)$ & $0.23(0.1)$ \\
\hline
\end{tabular}

CHR, change in heart rate. 
1. Age, record measured height and weight.

2. Do you play sport at present? Which sports?

3. How often do you play soccer (include practice) each week?

4. Which team do you play for?

5. Did you play football at school or for local teams $(\mathrm{Y} / \mathrm{N})$, which teams?

6. How often did you play football during your school years?

7. Did you play any other sport regularly while at school? Which ones?

8. Do you suffer from any medical conditions?

9. Have you ever had a serious injury while playing sport? (Y/N) Describe...

10. Have you ever suffered any injury to your legs or pelvis which resulted in you being hospitalized? (Y/N) Describe...

11. Have you ever had any problems with your knees? (Y/N) Describe...

12. Do you wear knee supports?

13. Have you ever consulted a doctor about a knee problem?

14. Have you ever received treatment for knee problems? (Y/N) Describe...

Figure 1 The questionnaire.

about symptoms such as locking and instability. They were also asked about their participation in sport to ensure that control subjects did not play soccer or any other field sports, and to ascertain how frequently soccer players participated and which teams they played for.

STATISTICAL ANALYSIS

Results are presented as means (SD). All normally distributed data were analysed using parametric statistical tests; Student's $t$-test was used for comparing two groups, and central tendency was illustrated using the mean value. All data that were not normally distributed were analysed using non-parametric tests, the difference between two groups was assessed using the Mann-Whitney U test (MWU), and central tendency was illustrated using the median value.

\section{Results}

The subjects consisted of 17 soccer players and 12 controls. Soccer players had a PCI of 0.42 (0.12) beats $/ \mathrm{m}$ and controls a PCI of 0.23 $(0.06)$ beats $/ \mathrm{m}$. These values are significantly different at the $\mathrm{p}=0.0001$ level (MWU).

The soccer players and controls were not significantly different in age or height (Student's $t$-test), but there was a significant difference in mass: soccer players had a mean mass of $75.6 \mathrm{~kg}$ and controls $67.3 \mathrm{~kg}(\mathrm{p}=0.04$; Student's $t$-test). Mass did not correlate with PCI $\left(r^{2}=-0.07\right)$; when soccer players and controls were weight-matched (nine subjects from each group matched within $2 \mathrm{~kg}$ ), there was still a significant difference in PCI values between the two groups ( $p=0.007$; MWU).

HEART RATES, WALKING SPEEDS, AND PCI

Table 1 gives heart rate, walking speed, and PCI values. There was no significant difference

Table 2 Reported knee problems and physiological cost index (PCI) (values for PCI are mean (SD))

\begin{tabular}{|c|c|c|c|c|c|c|}
\hline & \multicolumn{2}{|c|}{ All subjects $(n=29)$} & \multicolumn{2}{|c|}{ Soccer players $(n=17)$} & \multicolumn{2}{|c|}{ Controls $(n=12)$} \\
\hline & No & $P C I$ & No & $P C I$ & No & $P C I$ \\
\hline No problems & 12 & $0.25(0.1)$ & 3 & $0.34(0.05)$ & 9 & $0.22(0.06)$ \\
\hline Minor problems & 11 & $0.37(0.1)$ & 8 & $0.40(0.1)$ & 3 & $0.27(0.2)$ \\
\hline Major problems & 6 & $0.47(0.15)$ & 6 & $0.47(0.15)$ & 0 & Invalid \\
\hline
\end{tabular}

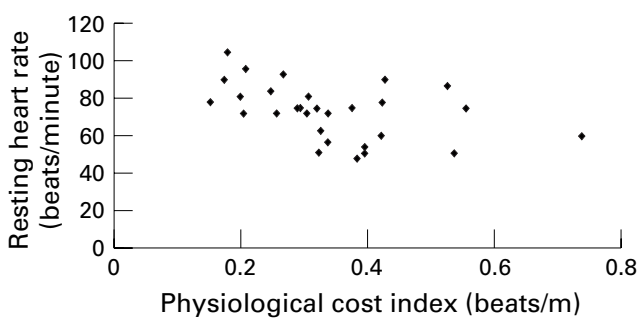

Figure 2 Relation between physiological cost index and resting heart rate.

in resting heart rate, walking heart rate, or walking speed between the two groups. There was a significant difference for the change in heart rate values $\left(\mathrm{p}=1.55 \times 10^{5}\right.$; Student's $t$-test), and this is the basis for the difference in PCI.

The relation between PCI and resting heart rate was investigated to see if fitness influenced PCI. Figure 2 is a scatter plot of PCI and resting heart rate; there is no significant relation$\operatorname{ship}\left(r^{2}=0.19\right)$.

\section{THE QUESTIONNAIRE}

Reported knee joint problems

All subjects $(n=29)$ were divided into three groups: those reporting no problems, those with minor problems and those with major problems. A problem was considered to be major if it had motivated the individual to seek medical advice. Table 2 gives the PCI values for the subgroups. For all subjects, the group with no problems had significantly lower PCI values than the group with minor problems and the group with major problems $(p=0.0025$ and $p$ $=0.0004$ respectively; Student's $t$-test). There was a much higher rate of knee problems in the group of soccer players; $82 \%$ reported problems compared with only $25 \%$ of controls.

\section{Participation in soccer and $P C I$}

Analysis of the data from soccer players showed that the current frequency of playing soccer and the quality of the team did not influence PCI values. However, participation in soccer while at school was shown to have significant effects.

All subjects ( $\mathrm{n}=29$ ) were asked how many times each week they played soccer at school. Thirteen subjects played less than four times a week and their mean PCI was 0.26 (0.09) beats $/ \mathrm{m} ; 16$ subjects played four or more times a week and their mean PCI was $0.41(0.12)$ beats $/ \mathrm{m}$ ( $\mathrm{p}=0.001$; Student's $t$-test). There was a small correlation between PCI value and the frequency of playing soccer at school $(r=$ $0.65)$.

The subjects were asked which teams they played for while they were at school. Ten did not play soccer at school (mean PCI $=0.24$ $(0.08)$ beats $/ \mathrm{m})$, nine played for the school team only (mean PCI $=0.33(0.08)$ beats $/ \mathrm{m}$ ) and ten played for the school team and one other team - for example, the county (mean PCI $=0.45(0.13)$ beats $/ \mathrm{m})$. All three groups were significantly different from each other $(\mathrm{p}<0.05)$. 


\section{Discussion}

The most interesting result from the present study is the significant difference in PCI values between the group of soccer players and the group of control subjects. The control group of 12 men aged 20-25 had a mean PCI value of $0.23(0.1)$ beats $/ \mathrm{m}$. Engsberg et al reported a mean value of $0.24(0.09)$ beats $/ \mathrm{m}$ for a control group of 13 healthy children aged 7-17 years walking on a treadmill at a self selected walking speed.

Other normal values in the literature are in fact considerably higher than those reported in this study. Barnett et $a l^{4}$ reported a median PCI value of 0.33 beats $/ \mathrm{m}$ in a group of 40 normal subjects aged 18-46 years, and Rose et al found a mean PCI of 0.47 beats $/ \mathrm{m}$ in a group of 102 normal children, aged 6-18 years. The selection criteria for normal subjects in these studies was less strict than those used in the present study, and the control group in the original Barnett study covered a wider age range. We would recommend that future studies of PCI should include only carefully selected controls. Control subjects should be questioned thoroughly about lower limb problems, and soccer players and possibly other regular sports players should be excluded from control groups.

The mean PCI value of $0.42(0.12)$ beats $/ \mathrm{m}$ obtained for soccer players is similar to the mean value of $0.41(0.18)$ beats/m obtained by Engsberg et $a l^{2}$ for below-knee amputee children walking with well fitted prosthetic limbs. It is lower than the PCI values obtained in this laboratory for adults with osteoarthritis or rheumatoid arthritis ${ }^{4}$ (medians used because the data were skewed were 0.53 and 0.935 beats $/ \mathrm{m}$ respectively). Other authors have reported PCI values for children with diplegic cerebral palsy ${ }^{3}$ (mean $1.46(0.12)$ beats $/ \mathrm{m}$ ) and paraplegics ambulating with assistive devices ${ }^{5}$ (mean 3.11 (1.14) beats/m, median 3.31 beats/ $\mathrm{m})$. The increasing mean or median PCI value for these different groups of patients corresponds to increasing degrees of functional impairment caused by the different pathologies.

The similarity between our results and those of Engsberg et al could be due to the fact that $90 \%$ of the Engsberg group were male, they were tested at their self selected walking speed, and they had a lower limb amputation but no other medical problems. We think the results are similar because the two groups have similar degrees of functional impairment reducing their walking efficiency. The PCI values reported by Engsberg's group reflects the quality of modern prosthetic devices and the children's ability to adapt to them; they all had been using prostheses for at least four years.

Engsberg et $a l^{2}$ found a significant correlation between PCI and oxygen consumption $\left(r^{2}\right.$ $=0.91)$ in both the below-knee amputee and control groups. He concluded "because PCI was significantly greater for below knee amputee children when compared to able bodied children it might be a useful tool to quantify differences between various groups of children". We think it is also useful for comparing groups of sportsmen; other possible uses are the assessment of training regimes and recovery from injury in individuals.

There are several possible reasons why soccer players have higher PCI values than control subjects who do not play soccer. Increased PCI values can be due to joint or soft tissue pathology and muscular or neuromuscular problems. Raised PCI values seen in patients with arthritic conditions are a result of decreased joint mobility and the presence of pain, and raised values seen in cerebral palsy and paraplegic patients result from paralysis or spasticity of one or more muscles. Neuromuscular dysfunction results in higher PCI values than joint and soft tissue pathology, as more active compensation is necessary to overcome this type of impairment.

The soccer players in our study may have a higher PCI than the controls because they are fitter; they do have a lower resting heart rate (but this is not significant). The soccer players and controls had the same walking speed and walking heart rate but the soccer players had a significantly higher PCI based on a higher change in heart rate (PCI $=$ (WHR RHR)/WS).

Fit individuals should have similar PCI values, as they should have a reduced heart rate at submaximal exercise intensities compared with unfit individuals ${ }^{6}$ as well as a lower resting heart rate, making the change in heart rate similar. The walking heart rate was the same in both groups; oxygen uptake at a given heart rate increases with training, ${ }^{6}$ and a high PCI corresponds to a high oxygen consumption as they have a positive linear relationship. ${ }^{2}{ }^{3}$ Therefore we can hypothesise that the soccer players have higher oxygen consumption for the same submaximal workload as the control group. Future studies could compare energy efficiency of gait in soccer players or groups with defined types of lower limb problems using methods based on the measurement of oxygen consumption.

The control subjects walked with a mean change in heart rate of 16 (3) beats/minute and walking speed of 66 (9) $\mathrm{m} /$ minute; the mean PCI value was 0.24 beats $/ \mathrm{m}$. The soccer players walked with an increased change in heart rate (mean 27 beats/minute) and a normal walking speed of $65 \mathrm{~m} /$ minute; their mean PCI value was 0.42 beats $/ \mathrm{m}$. Osteoarthritis patients in the study of Barnett et $a l^{4}$ walked with what we would consider to be a normal change in heart rate (median 18 beats/minute) but at relatively low walking speeds $(33 \mathrm{~m} /$ minute $)$ and had a median PCI of 0.53 beats $/ \mathrm{m}$. The patients with rheumatoid arthritis walked with a raised change in heart rate (median 27 beats/minute) and a slower walking speed $(29 \mathrm{~m} /$ minute $)$ and had a median PCI of 0.935 beats $/ \mathrm{m}$.

The osteoarthritis patients reduced their walking speed and this resulted in a higher energy expenditure for every metre walked, but the rate of energy expenditure (per minute) was normal, as the heart rate was not higher than that of normal controls. The raised PCI seen in osteoarthritis patients is entirely a result of their reduced walking speed. In contrast, the 
rheumatoid arthritis patients had a reduced walking speed and an increased heart rate, therefore both their energy expenditure per unit distance and rate of energy expenditure were increased; this probably reflects the systemic nature of rheumatoid arthritis. Our soccer players increased their rate of energy expenditure by increasing their change in heart rate but maintained their walking speed. The soccer players may have early degenerative joint disease which is compensated for in a different way from the osteoarthritis patients. However, we feel it is more likely to indicate that the soccer players have soft tissue damage in the lower limbs.

Ekstrand $e t a l^{7}$ found that soccer players had more muscular stiffness than non-soccer players and that most players had one or more tight muscles in the lower leg. If our soccer players had one or more tight muscles in the lower leg, this would raise their PCI as it would reduce the range of movement at the joints of the lower limb, and other muscles or muscle groups would have to compensate for the reduced ability to generate force in the tight muscle. Muscle tightness is a generally a result of poor training technique, especially the use of inadequate or incorrect muscle stretching exercises and inadequate warming down after training and matches. As this may produce temporary soft tissue problems, future studies could assess PCI in soccer players from teams that use good training techniques and test the players after a period of rest.

The group of soccer players $(n=17)$ had a very high rate of knee problems: six $(35 \%)$ reported major problems, eight (47\%) reported minor problems and only three $(18 \%)$ did not have problems. It is possible that repeated loading of the knee and injury during soccer in this subject group has led to changes in the cartilaginous structures of the knee similar to those reported by Wacker et al, ${ }^{8}$ which has resulted in the group having a raised PCI.

Wacker $e t a l^{8}$ examined the knee joints of 21 juvenile soccer players and 12 control subjects using magnetic resonance imaging. In all subjects the cruciate ligaments were normal. The articular cartilage was $24.8 \%$ thicker in the group of soccer players and increased signals in the centre of the menisci were observed in $38 \%(n=8)$ of the soccer players and $15 \%(n=2)$ of the controls. An increased amount of joint fluid was seen in $24 \%(n=5)$ of the soccer players but not in any of the controls. Increased signals in the centre of the menisci indicate meniscal degeneration; this would predispose the individual to meniscal tears and cartilage fibrillation. The increases in joint fluid and articular cartilage thickness observed in soccer players could be either a physiological response to external forces and torque, which increases the ability of the joint to absorb shock, or early signs of degeneration.

We found that those subjects who played soccer frequently and to a high standard when they were at school had higher PCI values than those who played infrequently. The subjects were put into three groups: those who played for the school team and another team, those who played for the school, and those who did not play for a team. The three groups had significantly different PCI values. This may be because those subjects who played a lot at school are more likely to have continued to play at college, and the raised PCI is due to recent temporary soft tissue damage. The subjects who played frequently in matches for good teams will have put more stress on their lower limbs and may have sustained more injuries as youths, which may have resulted in permanent soft tissue and joint damage.

\section{Conclusions}

University student soccer players have a higher energy cost for level walking than non-soccer players, which is accompanied by a high rate of reported knee joint problems.

This may be due to temporary or permanent injury to the skeleton or soft tissues, and may be related to poor training techniques and the effects of playing soccer frequently and for good teams when younger.

1 Inklaar H. Soccer injuries. I. Incidence and severity. Sports Med 1994;18:55-73.

2 Engsberg JR, Herbert LM, Grimston SK, et al. Relation among indices of effort and oxygen uptake in below-knee amputee and able-bodied children. Arch Phys Med Rehabil 1994;75:1335-41.

3 Rose J, Gamble JG, Burgos A, et al. Energy expenditure index of walking for normal children and for children with cerebral palsy. Dev Med Child Neurol 1990;32:333-40.

4 Barnett TG, Logan JG, Moore DJ, Paterson JM. Changes in heart rate of adults walking at their self selected speed. $\mathcal{F}$ Physiol (Lond) 1995;487:170P.

5 Nene AV, Jennings SJ. Physiological cost index of paraplegic locomotion using the ORLAU ParaWalker. Paraplegia 1992;30:246-52.

6 Raven PB, Hagan RD. Cardiovascular responses to exercise and training. In: Harries M, Williams C, Stantish WD, Micheli LJ, eds. Oxford textbook of sports medicine. Oxford: Oxford University Press, 1994

7 Ekstrand J, Gillquist J, Möller, et al. Incidence of soccer injuries and their relation to team success. Am F Sport Med 1983;11:63-7.

8 Wacker F, Konig H, Felsenberg D, Wolf KJ. MRI of the knee joint of young soccer players. Are there early changes of the internal structures of the knee due to competitive sports? Rofo Fortschr Geb Rontgenstr Neuen Bildgeb Verfahr 1994; 160:149-53. 